\title{
Analysing Mixed Reality Simulation for Industrial Applications: A Case Study in the Development of a Robotic Screw Remover System
}

\author{
Ian Yen-Hung Chen ${ }^{1}$, Bruce MacDonald ${ }^{1}$, Burkhard Wünsche ${ }^{1}$, Geoffrey \\ Biggs $^{2}$, and Tetsuo Kotoku ${ }^{2}$ \\ 1 University of Auckland, New Zealand \\ $\{$ i.chen, b.macdonald\}@auckland.ac.nz \\ burkhard@cs.auckland.ac.nz \\ 2 Intelligent Systems Research Institute, National Institute of Advanced Industrial \\ Science and Technology (AIST), Tsukuba, Japan \\ \{geoffrey.biggs, t.kotoku\}@aist.go.jp
}

\begin{abstract}
A Mixed Reality (MR) simulation aims to enable robot developers to create safe and close-to-real world environments from a mixture of real and virtual components for experimenting with robot systems. However, the reliability of the simulation results and its usefulness in solving practical problems remain to be validated. This paper presents an evaluation of an MR simulator by examining its use for the development of a robotic screw remover system. Quantitative evaluation compares the robot's trajectories produced in our MR simulation with those from a real world experiment, yielding results that indicate the MR simulation reliably represents the real world. A user study was conducted and the results demonstrate that the MR simulator gives users a stronger confidence of accurate results in comparison to a virtual simulator.
\end{abstract}

\section{Introduction}

While virtual simulation is useful for debugging and prototyping, it does not eliminate the need for eventual testing in the real world. A common problem faced is the discrepancies between the results produced in simulation and those from the actual operation. Mixed Reality (MR) simulation [5] is proposed to facilitate reliable transfer of simulation results to reality by bridging the gap between virtual simulation and real world tests. The simulator is founded on the concept of mixed reality [7] and enables developers to design a variety of scenarios for evaluating robot systems involving real and virtual components.

MR simulation can be considered as a form of hardware-in-the-loop simulation. In addition, it stresses the importance of seamless integration between the real and the virtual world to create a coherent environment for experimentation. Without strong coherency and sychronisation, such simulations could generate unnatural behaviour and produce inaccurate results that are unreliable than 
other forms of simulation. In our MR simulator, a server maintains a representation of the physical environment where the experimentation takes place. With a model of the real world available, interactions with objects in the virtual world can be simulated based on the framework described in [6].

Thorough evaluation of a simulation tool is important to extrapolate its use in practice. USARSim [3] is a virtual robot simulator that has undergone extensive validation. Efforts have been put into assessing the accuracy of its sensor, actuator, and environment models. However, similar efforts have not yet been applied to evaluation of MR/hybrid environments designed for robot development. While these experimentation methods exist, e.g. [9], the work has been limited to system design and implementations.

Equally important is the need for evaluating the visual interfaces of a simulator. A high fidelity robot simulator is of little use if the users cannot extract meaningful information from the simulation. The proposed MR simulator adopts Augmented Reality (AR) and Augmented Virtuality (AV) visualisation techniques which need to be evaluated on their effectiveness in conveying robot and context information to developers. Similar work was done by Nielsen et al. [8] who evaluated their 3D AV interface for assisting robot teleoperation tasks by comparing it with a traditional $2 \mathrm{D}$ interface. In contrast, we compare the $\mathrm{AR}$ and $\mathrm{AV}$ interfaces provided in our MR simulator to identify the strengths and weaknesses of each approach.

This paper presents an evaluation of MR simulation by case study. The goal is to assess the use of MR simulation for solving real world problems commonly faced during the robot development process. In this evaluation, an MR simulator is used to aid the development of a ceiling beam screw remover system to be deployed during the building demolition and renovation process. The remainder of the paper is organised as follows. Section 2 gives a background of the screw remover project. Section 3 describes the MR simulation created for the screw remover system. Section 4 presents results from quantitative evaluation. Section 5 describes the user study and its findings.

\section{Screw Remover Project}

In Japan, building interior renovation commonly requires removing equipments, such as lights and air conditioning vents, mounted on suspended beams of the ceiling. The old ceiling panels attached to the beams by self-tapping screws also need to be removed. A construction worker must then remove these screws by hand. This is a long and physically demanding process, due to the position of the screws above the worker's head. It is, however, simple and repetitive, making it ideal for automation. A ceiling beam screw removal robot [2] is proposed for the screw removal step. Rather than cutting or pulling the screws from the beam, which damages both the screws and the beam, the system uses a more sustainable solution, using a custom screw removal tool to unscrew the screw, leaving the materials in a reusable condition. The robot used is a Mitsubishi PA10 7 degree-of-freedom industrial robot manipulator. Mounted on the robot's 


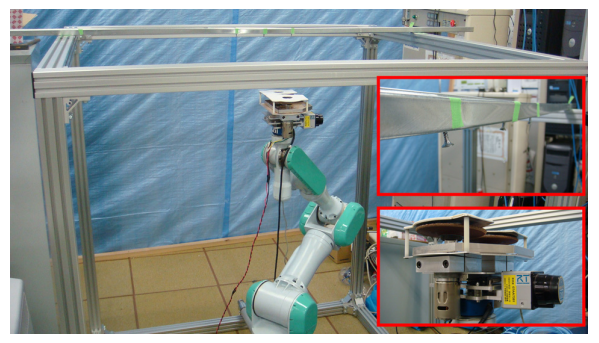

Fig. 1. A typical screw remover system test setup. Right: Ceiling beam and screw removal tool.

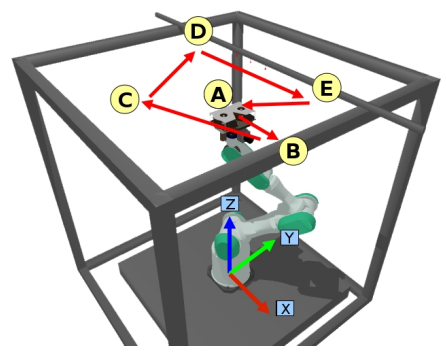

Fig. 2. Screw removal operations.

end-effector are a custom screw removal tool, a force-moment sensor, and a laser scanner (see Fig. 1). The custom screw remover tool consists of two rotating wheels that grip and turn the screw to remove it as the tool moves along the ceiling beam. The force-moment sensor is used to detect contact between the tool and the screws and in keeping the tool pressed against the beam. The laser is used to locate the beam above the robot and align the tool with the beam.

\subsection{Screw Removal Operations}

To perform the screw removal task, a human operator is required to press buttons on a Graphical User Interface (GUI) to start and stop the system. Once the operator starts the system, the robot will autonomously carry out the screw removal process. The operator monitors the robot operations and stops the system when the screws have been removed. The operations are shown in Fig. 2.

- A to B: The operator starts the system. The end-effector moves in the $x$-direction and takes three to five laser scans to locate the beam above.

- B to C: The end-effector moves to a position slightly below the beam.

- C to D: The end-effector approaches the beam at constant velocity until the force-moment sensor detects contact with the beam.

- D to E: The end-effector moves along the beam to remove screws.

- E to A: The operator stops the removal process and sets the robot to return to the starting position.

\section{Mixed Reality Simulation}

MR simulation integrates virtual resources into the real experimental setup to provide a cost-effective solution for experimenting with robot systems. The use of MR simulations can aid in the testing of the screw remover system because:

- There is a reasonable level of risk involved. The PA10 is a powerful robot and can cause harm to the human operator and the environment during 
development. Dangerous or expensive components can be virtualised in MR simulation to ensure safety.

- Testing of the system consumes resources such as spare beams and screws, which can be damaged by the robot due to errors in the system. The tool's wheels become worn out over time and need to be replaced. MR simulation is able to minimise the resources consumed by simulating these materials.

In this MR simulation, we are interested in studying the behaviour of the robot in the real world, making sure the controllers are generating the correct movements before moving onto a completely real world test. We would also like to minimise resource requirements in the testing process. Therefore we chose to virtualise the ceiling beam and the screws. The components in this setup are:

Real: robot manipulator, screw removal tool, laser sensor, force-moment sensor, and frame (for holding the ceiling beam).

Virtual: a ceiling beam fitted with three screws.

It is important that in an MR simulation, real and virtual objects are able to seamlessly interact with one another as if they exist in a coherent environment. To facilitate interaction between the real robot components and the virtual beam and screws, the sensors and actuators of the screw remover system need to be augmented with virtual information. The real laser needs to detect the location of the virtual beam as well as the real frame, while the real force-moment sensor needs to detect contact between the screw removal tool and the virtual beam as well as other external forces in the real world. The motion of the real robot manipulator will also be influenced by friction between the real screw removal tool and the virtual beam, and contacts between the screw removal tool and the virtual screws. Interaction between real and virtual components has been created based on the framework proposed in [6]. Exact simulation of the actual screw removal process (wheels gripping and turning the screw) is considered unnecessary for this application as we are interested in simulation at an integration level. Thus, the screw removal process is simplified at the cost of lower simulation accuracy. In our simulation, once the screw has a force exerted on it exceeding a threshold over a certain amount of time, the screw falls from the beam.

To create an MR simulation for the screw remover system that runs on the OpenRTM framework [1], the MR simulator described in [5] has been enhanced with the support of simulating OpenRTM-based systems, and the simulator is now based on the Gazebo simulation environment for OpenRTM [4]. OpenRTMaist is a distributed, component-based framework. Each part of the system is implemented as a separate software component. These components communicate over known interfaces. Fig. 3 shows the system diagram of the components in the MR simulation. The screw remover system now exchanges data with MR sensors and an MR robot manipulator, instead of reading data from a real laser or force-moment sensor and sending commands to the real PA10. In summary, MR sensors make use of the real sensor components, modify their raw data with inputs from the virtual world created using Gazebo, before publishing to the connecting components. The MR robot manipulator models any physical contacts with virtual objects then sends commands to move the real PA10 accordingly. 


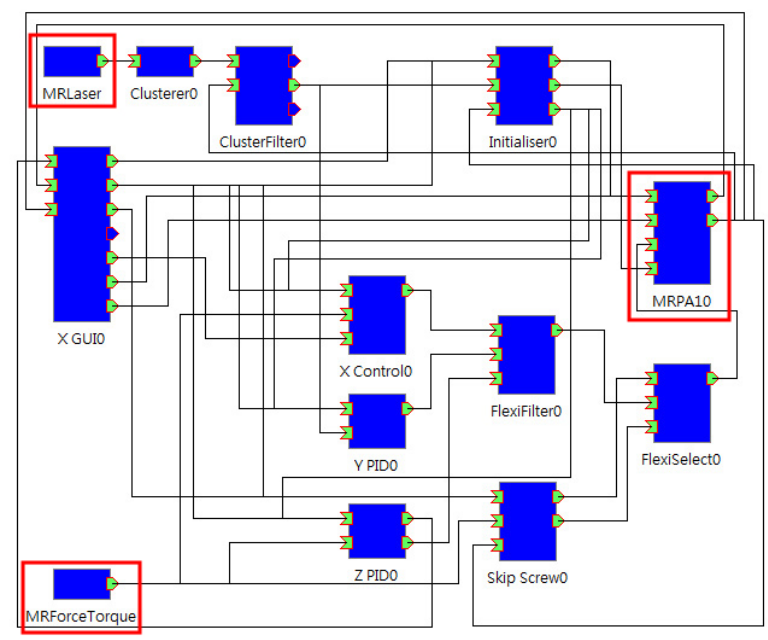

Fig. 3. System diagram of components in the MR simulation. The components highlighted in red are MR sensors (MRLaser, and MRForceTorque) and an MR robot manipulator (MRPA10) that exchange data with the original screw remover system.

\subsection{MR Interfaces}

The AR and the AV interface provided are shown in Fig. 4. In the AR interface, virtual objects are overlaid in geometric registration onto the real world scene using a markerless AR technique that tracks point features in the scene to compute the camera pose. A fixed overhead camera is used to capture a view of the whole experimentation environment which enables the users to monitor the overall robot manipulator's movement and the screw removal process.

The AV interface provides a view in a virtual environment augmented with real world information. It is designed based on the ecological interface paradigm [8] that integrates multiple sensor information in an integrated display. A free-look camera is available in the AV interface that can be controlled to move freely within the environment for observing the simulation.

\section{Quantitative Evaluation}

An experiment was conducted to compare results obtained from the MR simulation and a real experiment. Each experiment was run five times, and the trajectories taken by the end-effector of the robot manipulator recorded. The time for a single screw removal was set to be 6 seconds in the MR simulation.

The average trajectories are shown in Fig. 5. One thousand evenly spaced sample points were taken from each trajectory to calculate the averages. The results show that the average path taken by the robot in the MR simulation closely resembles the one in the real experiment. This indicates that the registration of the virtual beam and screws in the real world is accurate and the 


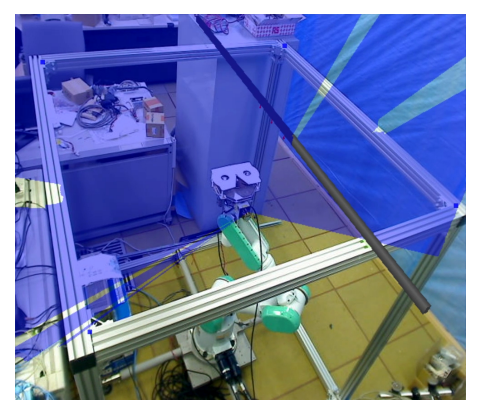

(a)

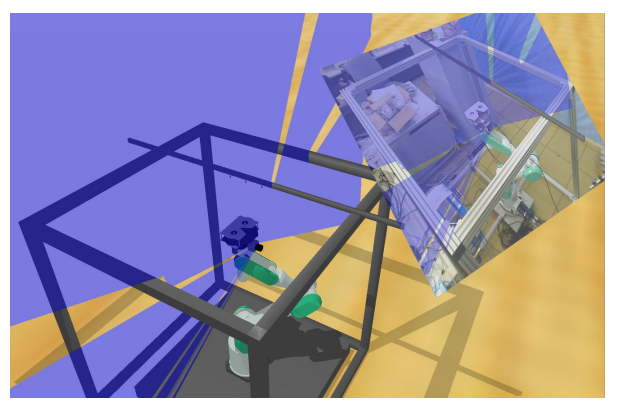

(b)

Fig. 4. Screenshots of MR interfaces: a) AR interface showing visualisation of laser data, virtual beam, and virtual screws in context with the real world, b) AV interface showing the simulation in a virtual environment augmented with real world laser and camera data.

robot successfully detected these virtual objects. Note that the deviation in the last segments of the trajectories between MR simulation and the real experiment are caused by the inconsistency of the human operator when signaling the robot to stop the screw removal operation and return to the starting position. The Root Mean Square Error (RMSE) between the two average trajectories was calculated by comparing the distances between the corresponding sample points from the two paths, and is found to be $18.08 \mathrm{~mm}$. The RMSE is approximately $1.10 \%$ with respect to the average distances travelled by the end-effector, which is $1621.79 \mathrm{~mm}$ in MR simulation, and $1643.79 \mathrm{~mm}$ in the real experiment.

Fig. 6 shows the trajectories over time for the five runs. As the robot's endeffector moves along the beam in the $x$-direction to remove screws, a step-like pattern can be observed. The results clearly show that more variations occur in the real experiment which the MR simulation is unable to produce due to the simplified screw removal process and the fixed screw removal time. Table 1 shows the average times for removing a single screw, and for completing the entire task. The average time for removing a single screw in the real experiment is shorter than predicted, resulting in a short task completion time compared to the MR simulation.

\section{User Study}

A within-subject user study was conducted to compare the user's experience in using MR simulation with a purely virtual simulator for performing a robotic task. Each participant carried out the task twice: once in the virtual simulation, and once in the MR simulation. To minimise any learning effects, some participants carried out the task in virtual simulation first, while some started with MR simulation. 

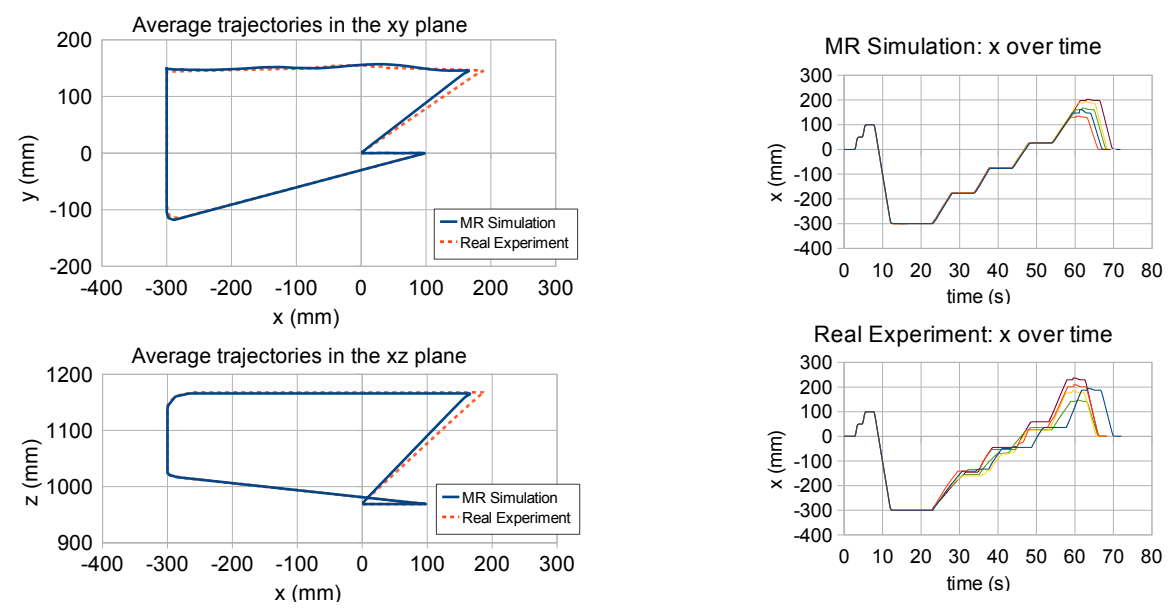

Fig. 5. Comparison between the average trajectories of the robot's end-effector in $x y$ - and $x z$-plane.

Fig. 6. Raw trajectories of the robot's end-effector over time

Table 1. Average Times

\begin{tabular}{lcc}
\hline & Screw Removal Task Completion \\
\hline MR Simulation & $6.00 \mathrm{~s} / \mathrm{screw}$ & $69.97 \mathrm{~s}$ \\
Real Experiment & $4.90 \mathrm{~s} / \mathrm{screw}$ & $68.95 \mathrm{~s}$ \\
\hline
\end{tabular}

In virtual simulation, the participants focused on a single virtual interface for carrying out the specified task. In comparison, MR simulation provides two interfaces: the AR interface and the AV interface. The participants were free to switch between the two interfaces provided for completing the task in MR simulation. The user's experience in using the two provided interfaces was also compared. Desktop videos were taken to examine the participants' actions and use of the provided interfaces when carrying out the task.

\subsection{Task}

The participants played the role of the human operator in the screw removal task outlined in Section 2.1. They were required to operate the screw removal robot and monitor its operation to ensure the robot completed its task correctly. The participants were first told about the screw remover project and the procedure for removing screws using the PA10 robot. The procedure involved the user clicking on buttons on a GUI for 1) initialising the system, 2) starting the screw removal operation, 3) stopping the screw removal operation, and 4) returning the robot to the starting position. 
The participants were then introduced to the MR simulator and the virtual simulator, Gazebo. They were given the opportunity to familiarise themselves with the keyboard and mouse controls of Gazebo that move a free-look camera for observing the simulation environment. Note that the same free-look camera was also available for them to use in the AV interface of MR simulation.

The operation of the screw removal task in virtual simulation was straight forward, with the participants using the GUI and the free-look camera for completing the task. In MR simulation, the participants were asked to perform an extra step that merges the real world and the virtual world. On the live video imagery captured by the camera overlooking the physical experiment environment, the participants needed to click with a mouse on the four corners of the frame that holds the ceiling beam. This calibrated the camera and registered the virtual objects into the real world for setting up the AR interface. The participants could then switch between the AR interface and the AV interface using the keyboard.

After completing the task using both simulation methods, the participants were asked to fill a questionnaire, followed by a short interview investigating any problems that they encountered when using the two simulators.

\subsection{Dependent Variables}

The time for task completion was recorded. The task completion time includes the time taken to position the free-look camera before operating the robot, and any time taken by the participant to observe the simulation before confirming the completion of the operation. The task completion time excludes the time taken to set up the AR interface in MR simulation (users manually clicking on four corners for calibrating the camera), which was recorded separately.

The time spent on each visual interface in MR simulation was also recorded to investigate if there are any preferences in using the provided interfaces for this specific task.

Paired t-tests are used to identify any significant differences between the times in this repeated-measures user study. A p-value smaller than 0.05 suggests the difference is significant.

\subsection{Questionnaire}

The questionnaire comprised three sections. Section one collected the participant's demographic information. Section two measured the participant's experience in using virtual simulation and MR simulation. Section three measured the user's experience in using the AR interface and the AV interface for carrying out the specified task. A 7-point Likert scale was used throughout the questionnaire for measuring the user's experience.

Two-tailed Wilcoxon Signed Rank tests are used to identify any significant differences between the users' experiences in using virtual simulation and MR simulation, and similarly, the users' experiences in using the AR interface and the AV interface. 


\subsection{Hypotheses}

1. Virtual simulation offers a safer environment than MR simulation for robot development.

2. MR simulation is a more accurate representation of the real world than virtual simulation.

3. MR simulation produces more reliable results than virtual simulation.

4. The AR interface provides a more intuitive display of information than the AV interface

5. The AV interface provides a more flexible view of the environment than the AR interface

6. The AV interface increases the user's awareness of the robot status and potentially dangerous situations.

\subsection{User Study Results}

11 participants were recruited for this study. Out of the 11 participants, 10 participants had experience in computer programming and/or robot development (Computer programming: 9 participants with mean 14.10 and Standard Deviation (SD) 6.12 years of experience; Robot development: 8 participants with mean 6.83 and SD 6.85 years of experience) and 8 had used a simulation tool before for the development of computer/engineering systems. One participant had no experience in computer programming or robot development.

6 participants were randomly assigned to carry out the task in virtual simulation first, while 5 participants began with MR simulation.

Simulation Experience The users completed the task faster in virtual simulation (mean 90.73 seconds, SD 13.75) than in MR simulation (mean 102.00 seconds, SD 23.21). Although the difference is not significant $(\mathrm{t}=-1.49, \mathrm{p}=$ 0.17 ), it was observed that the participants were being more cautious in MR simulation since a real robot was used, taking more time to observe the simulation environment through the interfaces provided and keeping an eye on the robot in the real environment throughout the task.

The questionnaire results on simulation experience are shown in Fig. 7. The users rated MR simulation significantly higher than virtual simulation for debugging $(\mathrm{Z}=-2.33, \mathrm{p}<0.05)$. The high rating is believed to be leveraged by the use of the AR interface in MR simulation, which helped users understand complex robot data.

The questionnaire results suggest that virtual simulation is a safer method than MR simulation and helps to minimise any potential harm. Despite the finding being statistically insignificant $(\mathrm{Z}=-0.96, \mathrm{p}=0.33)$, using a real robot in MR simulation does have an impact on the safety of the experiment. In the later interview we found that 4 participants expressed concern about the robot colliding with other real world objects in the environment, such as the ceiling beam frame, and 3 of whom reported being nervous while the screw removal operation was taking place in MR simulation. 


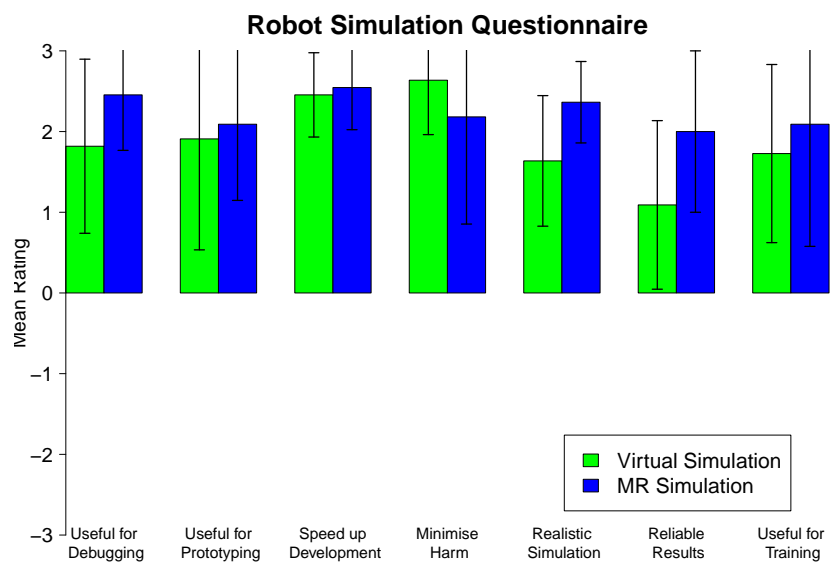

Fig. 7. Differences between the users' experiences in using virtual simulation and MR simulation.

MR simulation was rated significantly higher than virtual simulation for producing a more realistic simulation $(\mathrm{Z}=-2.06, \mathrm{p}<0.05)$, and more reliable results $(\mathrm{Z}=-2.46, \mathrm{p}<0.05)$, hence accepting hypothesis 2 and 3 . The finding is particularly encouraging as it indicates that MR simulation gave users a stronger confidence of accurate results, potentially allowing the simulator to be used for extended testing and evaluation of robot systems beyond the prototyping stage.

MR simulation was rated higher than virtual simulation for prototyping ( $\mathrm{Z}$ $=-0.53, \mathrm{p}=0.60)$, speeding up development $(\mathrm{Z}=-0.58, \mathrm{p}=0.56)$, and teaching and training $(\mathrm{Z}=-0.97, \mathrm{p}=0.33)$, but the differences are not significant.

MR Interface Experience On average, the user focused more on the AR interface (mean 61.73 seconds, SD 26.18) than the AV interface (mean 40.27 seconds, SD 22.64) for carrying out the screw removal task, but the difference is not significant $(\mathrm{t}=1.65, \mathrm{p}=0.13)$. The mean time for setting up the AR interface before starting the task in MR simulation is found to be 14.73 seconds (SD 5.59); the extra time is an overhead for using MR simulation and needs to be minimised in the future.

The questionnaire results on MR interface experience are shown in Fig. 8. The users rated the AR interface significantly higher than the AV interface for intuitive display of information $(\mathrm{Z}=-2.07, \mathrm{p}<0.05)$, and also commented that overlaying virtual robot data in the physical world made understanding robot data and debugging intuitive. Similarly, MR simulation also received a higher mean rating for learning robot data $(\mathrm{Z}=-1.93, \mathrm{p}=0.05)$, and identifying inconsistencies between the user's view and the robot's view of the world ( $\mathrm{Z}=$ $-1.63 \mathrm{p}=0.10$ ), although not statistically significant at the $\mathrm{p}<0.05$ level, these two attributes are believed to be strengths of $\mathrm{AR}$ visualisation. 


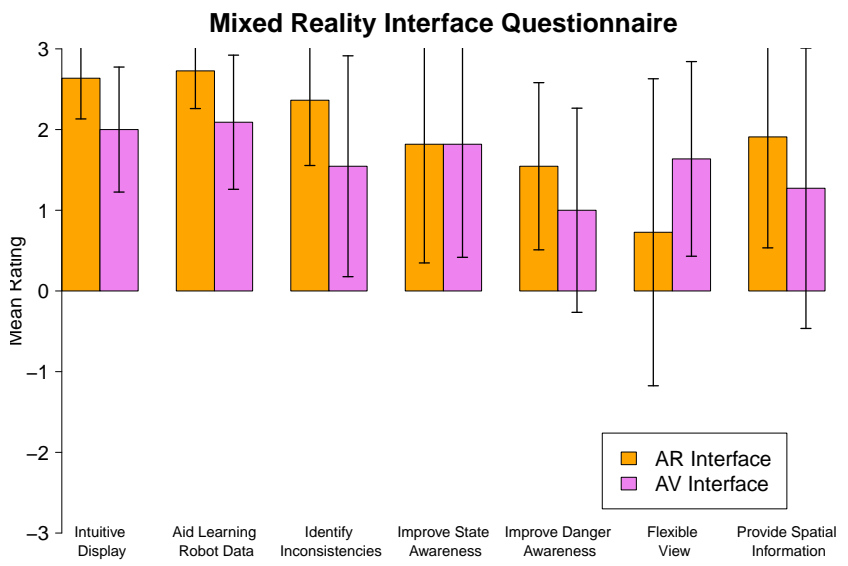

Fig. 8. Differences between the users' experiences in using the AR interface and the AV interface.

The users rated the AV interface higher for providing a more flexible view than AR interface, as expected, but the difference is not significant $(\mathrm{Z}=-1.73$, $\mathrm{p}$ $=0.08)$. However, a number of participants commented that they would prefer having the ability to move the physical camera around while using the AR interface rather than using a fixed camera. The results could differ if an on-board or movable camera was used. This needs to be further investigated.

Interestingly, a number of observations were not expected. The AR interface was rated equally important as the AV interface for improving awareness of robot states, and the AR interface was rated higher for improving awareness of dangerous situations $(\mathrm{Z}=-1.19, \mathrm{p}=0.24)$. This rejects hypothesis 6 . Furthermore, the users rated the AR interface significantly higher for providing spatial information than the AV interface $(\mathrm{Z}=-2.33, \mathrm{p}<0.05)$. This was a surprise as the AR interface is only a $2 \mathrm{D}$ display of information.

Overall, the users rated in favour of the AR interface. The results are believed to be influenced by the user's unfamiliarity with the control of the free-look camera in the AV interface. Although the users were given time to practice until they were comfortable, the later interview found that 5 participants (45\%) commented on the difficulty of using keyboard and mouse inputs in the AV interface. Suggestions include using a 3D mouse or a joystick as an alternative input device. Reviewing the recorded desktop videos discovered that many participants did not continue to move the free-look camera after the screw removal operation started. The free-look camera was left at a fixed position either looking over the entire simulation environment or zoomed in at the three screws to be removed. Two participants became lost in the AV interface and immediately switched to the AR interface to continue monitoring the screw removal operation. This finding suggests future work is necessary to improve the user input interface in order to exploit the full potential of the AV interface. 


\section{Conclusions and Future Work}

While previous research has demonstrated various ideas for MR/hybrid simulations, we have for the first time presented an evaluation of an MR robot simulator in an industrial application. An MR simulation has been created for a screw remover project. Comparative evaluation shows an RMSE of approximately $1.10 \%$ between the average trajectories taken by the robot in the MR simulation and the real experiment. User study results reveal that users are positive about MR simulation and believe it offers a more reliable transfer of results to reality. However, the visual interfaces need improvements. The AR interface helped users understand and debug robot data but provided a limited view of the scene; a movable viewpoint needs to be considered in the future. The AV interface lacks an intuitive user input interface for observing the simulation environment; more natural interaction methods are necessary to improve the usability of the system.

Acknowledgments. The research was financially supported by the National Institute of Advanced Industrial Science and Technology, Japan, and the University of Auckland Doctoral Scholarship.

\section{References}

1. Ando, N., Suehiro, T., Kotoku, T.: A software platform for component based rtsystem development: OpenRTM-aist. In: Proceedings of the 1st International Conference on Simulation, Modeling, and Programming for Autonomous Robots. SIMPAR'08. pp. 87-98. Springer-Verlag, Berlin, Heidelberg (2008)

2. Biggs, G., Kotoku, T., Tanikawa, T.: Ceiling beam screw removal using a robotic manipulator. In: Proceedings of the 2009 IEEE/RSJ International Conference on Intelligent Robots and Systems. St. Louis, USA (October 11-15 2009)

3. Carpin, S., T. Stoyanov, Y.N., Lewis, M., Wang, J.: Quantitative assessments of USARSim accuracy. In: Proceedings of PerMIS 2006 (2006)

4. Chen, I., MacDonald, B., Wünsche, B., Biggs, G., Kotoku, T.: A simulation environment for OpenRTM-aist. In: Proceedings of the IEEE International Symposium on System Integration. pp. 113-117. Tokyo, Japan (November 29 2009)

5. Chen, I.Y.H., MacDonald, B., Wünsche, B.: Mixed reality simulation for mobile robots. In: Proceedings of the IEEE International Conference on Robotics and Automation, 2009. ICRA'09. pp. 232-237. Kobe, Japan (May 12-17 2009)

6. Chen, I.Y.H., MacDonald, B.A., Wünsche, B.C.: Designing a mixed reality framework for enriching interactions in robot simulation. In: Proceedings of the International Conference on Computer Graphics Theory and Applications. pp. 331-338. Angers, France (May 17-21 2010)

7. Milgram, P., Colquhoun, H.: A taxonomy of real and virtual world display integration. Mixed Reality-Merging Real and Virtual Worlds pp. 5-28 (1999)

8. Nielsen, C., Goodrich, M., Ricks, R.: Ecological interfaces for improving mobile robot teleoperation. IEEE Transactions on Robotics 23(5), 927-941 (2007)

9. Nishiwaki, K., Kobayashi, K., Uchiyama, S., Yamamoto, H., Kagami, S.: Mixed reality environment for autonomous robot development. In: Proceedings of the IEEE International Conference on Robotics and Automation. pp. 2211-2212. Pasadena, CA, USA (May 19-23 2008) 